\title{
Long-range Kondo signature of a single magnetic impurity
}

\author{
Henning Prüser ${ }^{1}$, Martin Wenderoth ${ }^{1 \star}$, Piet E. Dargel ${ }^{2}$, Alexander Weismann ${ }^{1}$, Robert Peters ${ }^{2}$, \\ Thomas Pruschke ${ }^{2}$ and Rainer G. Ulbrich ${ }^{1}$
}

\begin{abstract}
The Kondo effect, one of the first recognized correlation phenomena in condensed-matter physics ${ }^{1}$, has regained attention because of scanning tunnelling spectroscopy (STS) experiments carried out on single magnetic impurities ${ }^{2,3}$. Despite the subnanometre resolution capability of local probe techniques, one of the fundamental aspects of Kondo physics, its spatial extension, is still subject to discussion. Until now all STS studies on single adsorbed atoms have shown that observable Kondo features vanish rapidly with increasing distance from the impurity ${ }^{4-9}$. Here we report on a hitherto unobserved long-range Kondo signature for single magnetic atoms of Fe and $\mathrm{Co}_{0}$ buried under a $\mathrm{Cu}(100)$ surface. We present a theoretical interpretation of the measured signatures using a combined approach of band-structure and many-body numerical renormalization group calculations. These are in excellent agreement with the rich spatially and spectroscopically resolved experimental data.
\end{abstract}

The interaction of a single magnetic impurity with the surrounding electron gas of a non-magnetic metal leads to fascinating phenomena in the low-temperature limit, which are encompassed by the term Kondo effect ${ }^{1}$. Such an impurity has a localized spin moment that interacts with the electrons of the conduction band. If the system is cooled below a characteristic temperature, the Kondo temperature $T_{\mathrm{K}}$, a correlated electronic state develops and the impurity spin is screened. The most prominent fingerprint of this many-body singlet state is a narrow resonance at the Fermi energy $\varepsilon_{\mathrm{F}}$ in the single-particle spectrum of the impurity, called Kondo or Abrikosov-Suhl resonance. The existence of this Kondo resonance has been experimentally confirmed for dense systems with high-resolution photoemission electron spectroscopy and inverse photoemission ${ }^{10,11}$. As a result of their limited spatial resolution these measurements always probe a very large ensemble of magnetic atoms. With its capability to study local electronic properties with high spatial and energetic resolution, STS has paved the way to access individual impurities ${ }^{2,3}$.

A theoretical prediction for the local density of states (LDOS) the key quantity measured in STS experiments-was first provided by Ujsághy and colleagues ${ }^{12}$. According to their calculations, the Kondo resonance induces strong spectroscopic signatures at the Fermi energy, with line shapes that are oscillatory with distance to the impurity. Since the first STS studies in 1998 (refs 2,3) many experiments on magnetic atoms and molecules on metal surfaces have been carried out, all of which show Kondo fingerprints ${ }^{5-9}$. However, it is worth noting that all previous STS experiments on isolated adatoms have reported that the Kondo signature vanishes rapidly within a few angstroms and no variation of the line shape occurs. Only when the electron's amplitude was amplified by placing the Kondo atom within an elliptical resonator structure could the Kondo signature of a Co atom be probed at larger distance $^{4}$. In this quantum corral experiment Manoharan et al. detected one resonance on the adatom and another localized signature in the second focus of the ellipse. However, no line shape variations with distance were reported (for a review on adatom Kondo systems see ref. 13).

In this work we follow a new route and investigate single isolated magnetic impurities buried below the surface with a low-temperature STM operating at $6 \mathrm{~K}$. It has recently been shown that the anisotropy of the copper Fermi surface leads to a strong directional propagation of quasi-particles, which is called electron focusing ${ }^{14}$. This effect gives access to individual bulk impurities in a metal that were previously assumed to be 'invisible' because of charge screening. Following these observations, dilute magnetic alloys were prepared on a clean $\mathrm{Cu}(100)$ single crystal by adding a small amount $(0.02 \%)$ of either Co or Fe to the topmost monolayers. We have chosen Co and Fe because of their different Kondo temperature. This allows the universal character of the Kondo effect to be tested. The actual layer depth of the impurities was determined by the size of the impurity pattern and by comparison to theoretical calculations $^{14,15}$ (see Supplementary Information).

As a first striking example of how the Kondo effect influences the energy-dependent scattering behaviour on the millivolt scale, Fig. 1a shows STM topographies of a fourth-layer Fe impurity for different bias voltages $V$ near zero millivolts. The local minimum of the LDOS present in the centre of the interference pattern for $V<0$ develops into a plateau-like maximum for $V>0$. In Fig. $1 \mathrm{~b}$ the differential conductance as a function of bias voltage and one spatial coordinate $y$ across the impurity pattern is shown. The crossover observed in the topographies occurs very close to zero bias. In Fig. 1c, four spectra for different positions A-D are shown, illustrating very clearly that a single Kondo atom buried below the surface of copper induces long-range spectral signatures that depend on the distance to the impurity. A second possibility for investigating the Kondo effect versus distance is to look at impurities situated at different depths $d$ below the surface. Figure 2 shows, as an example, STS data of Co atoms, comparing single spectra (purple curves) measured directly above the impurities $(y=0)$. The lateral variations of $\mathrm{d} I / \mathrm{d} V$ are again depicted as sections (upper part of Fig. 2). All show a constriction of the pattern for positive bias voltages. The comparison between $\mathrm{Co}$ and $\mathrm{Fe}$ data demonstrates that both impurity species show similar behaviour on completely different energy scales (for instance compare the fourth-layer Fe in Fig. 1b with a fourth-layer Co in Fig. 2).

For a quantitative analysis, we have to use advanced tools of quantum many-particle theory, as the Kondo problem is a genuine

${ }^{1}$ IV. Physikalisches Institut, Universität Göttingen, Friedrich Hund Platz 1, 37077 Göttingen, Germany, ${ }^{2}$ Institut für Theoretische Physik, Universität Göttingen, Friedrich Hund Platz 1, 37077 Göttingen, Germany. *e-mail: wenderoth@ph4.physik.uni-goettingen.de. 
a

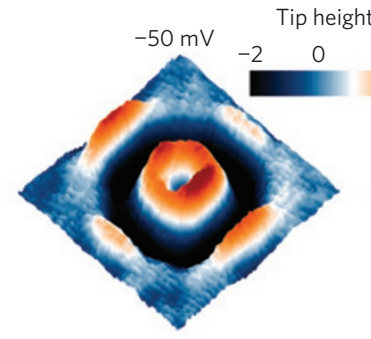

b

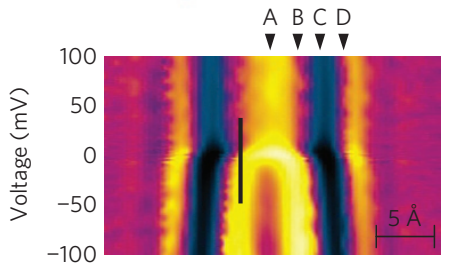

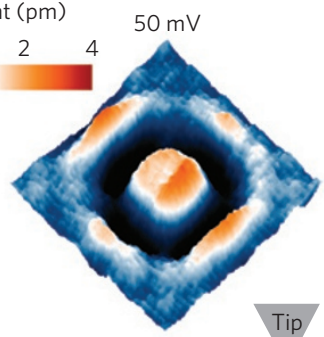

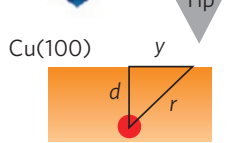

$\Delta \mathrm{d} l / \mathrm{d} V(\mathrm{pA} \mathrm{mV}-1)$

$-1 \quad 0 \quad 1$
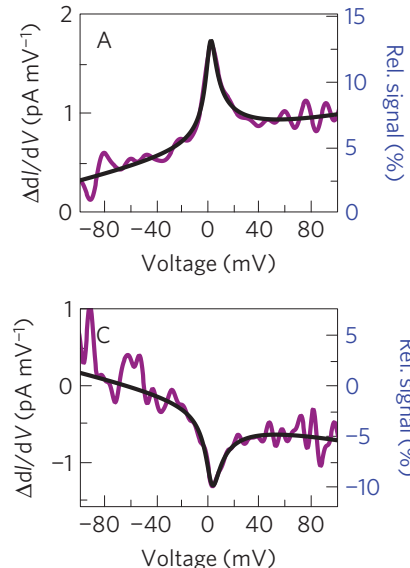
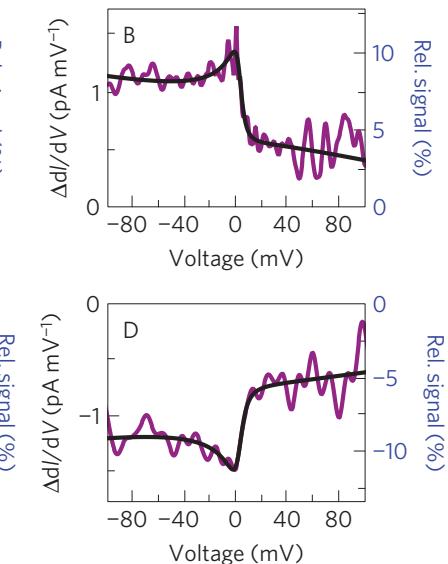

Figure 1 | Variation of the LDOS with the lateral distance of the tip. a, STM constant current topography $(1.8 \mathrm{~nm} \times 1.8 \mathrm{~nm}, 2 \mathrm{nA})$ of a fourth-layer Fe impurity for different bias voltages. For negative bias voltage the LDOS directly above the impurity is reduced. By increasing the voltage the central ring contracts to a plateau-like maximum and the lateral extension of the focusing pattern decreases. $\mathbf{b}$, Section of the differential conductance $\Delta \mathrm{d} l / \mathrm{d} V=\mathrm{d} l / \mathrm{d} V-\mathrm{d} l_{0} / \mathrm{d} V$ along the [010] direction. The tip height was adjusted to give a differential conductance for the free surface $\mathrm{d} l_{0} / \mathrm{d} V$ of $13.3 \mathrm{pA} \mathrm{mV}^{-1}$. An energy-dependent phase shift of the interference pattern can be observed. The vertical black line would resemble the phase front for a energy-independent scattering phase and indicates that the overall phase shift caused by the resonance is less than $\pi$. c, Single spectra (purple curve) for four different lateral distances A-D (marked in $\mathbf{b}$ by black arrows). To illustrate the relative amplitude of the Kondo signal, the data is normalized to the differential conductance of the free surface (blue scale on the right side). Black curve: calculated spectra obtained by fitting a phenomenological expression of the Kondo resonance to the differential conductance (see Supplementary Information).
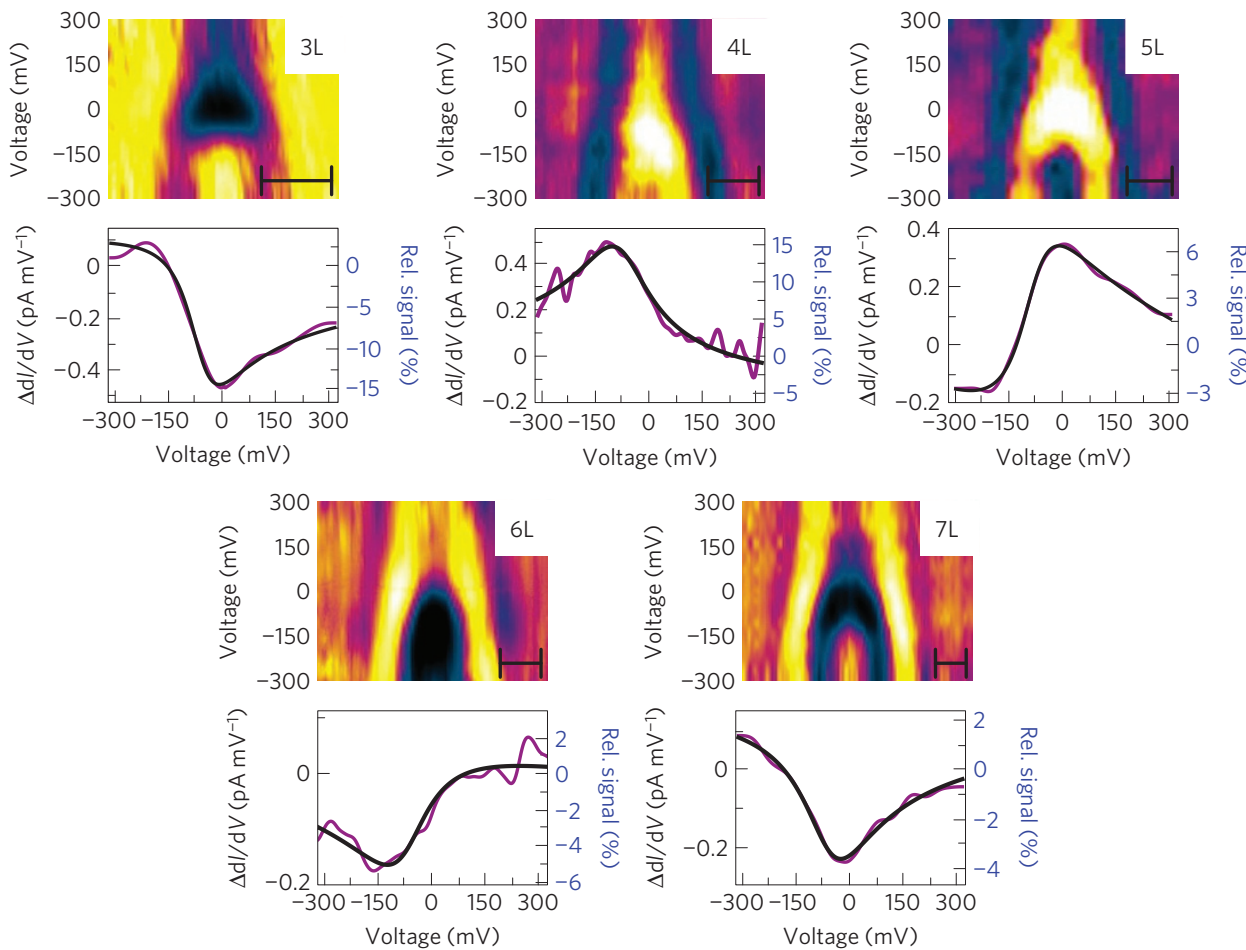

Figure 2 | STS data of subsurface Co impurities in three-seven layers below the Cu(100) surface. Spectrum sections $\Delta d / / d V=d l / d V-d / 0 / d V$ along the [010] direction are shown in the upper part. A strong change in the scattering behaviour can be seen for all impurity depths. The black bar corresponds to a length of $0.5 \mathrm{~nm}$ for every section. Single $\Delta \mathrm{dl} / \mathrm{dV}$ spectra (purple curve) measured with a tip position direct above the impurity $(d=3-7 \mathrm{ML})$ are depicted in the lower part. The tip height was adjusted to give a differential conductance for the free surface $\mathrm{d} / 0 / \mathrm{dV}$ of $3.2 \mathrm{pA} \mathrm{mV}-1\left(3,4,6 \mathrm{ML}^{-1}\right), 5.3 \mathrm{pA} \mathrm{mV}-1$ $(5 \mathrm{ML})$ and $6.4 \mathrm{pA} \mathrm{mV}^{-1}(7 \mathrm{ML})$. To illustrate the relative amplitude of the Kondo signal, the data are normalized to the differential conductance of the free surface (blue scale on the right side). Black curves: calculated spectra obtained by fitting a phenomenological expression of the Kondo resonance to the differential conductance (see Supplementary Information). Note that the colour scale for the spectroscopic data is similar to that used in Fig. 1, except the range varies, and is set by the corresponding single spectra.

many-body effect. The well-known universal behaviour of the Kondo effect and its associated low-energy fingerprints allows one to apply the single-impurity Anderson model. Within this model the localized orbital of the impurity is described by a single level 

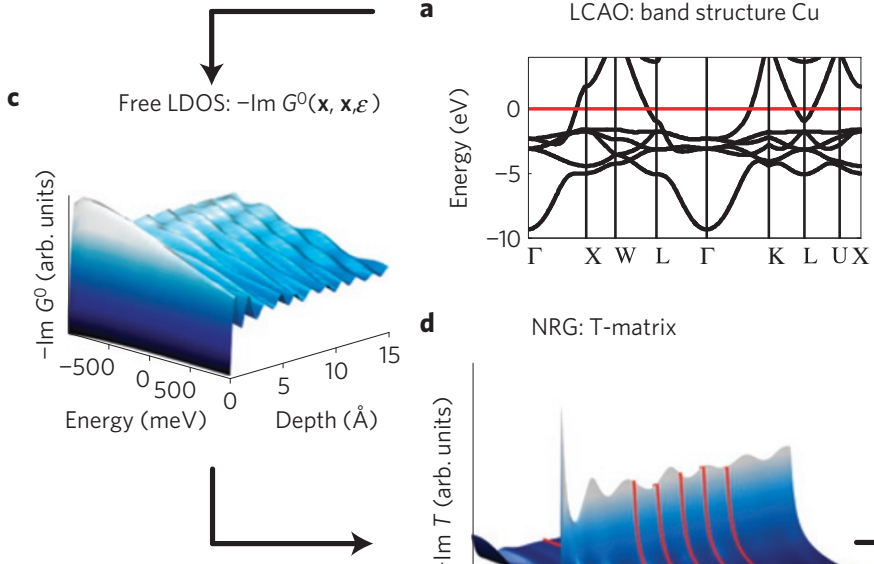

d NRG: T-matrix

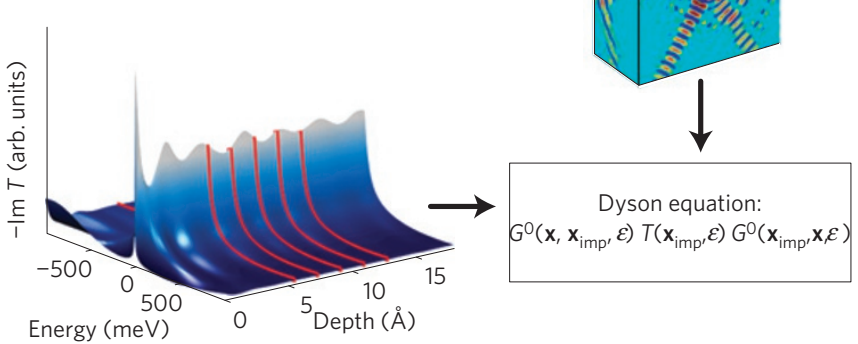

e $\quad \Delta$ LDOS section along [010] direction
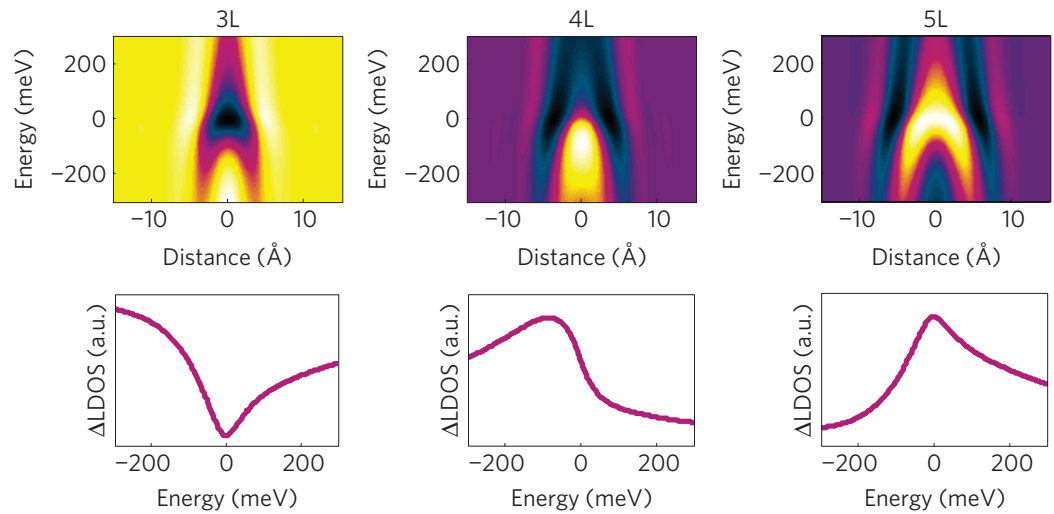

$6 \mathrm{~L}$
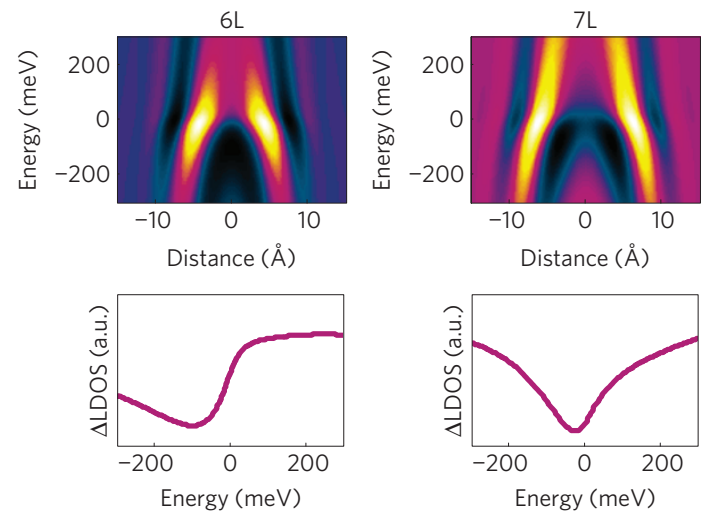

Figure 3 | Road map of the theoretical model. a, Band structure of copper calculated using LCAO and $\mathbf{b}$, the related free propagator. c, Unperturbed LDOS as function of depth, including effects of the surface. $\mathbf{d}$, The Kondo resonance for impurities located at different positions. $\mathbf{e}$, Calculated $\Delta$ LDOS section along the [010] direction for Co impurities situated 3-7 ML below the surface. Single spectra taken directly above the impurity position are shown below. Note that the colour scale for the spectroscopic data is similar to that used in Fig. 1, except the range varies, and is set by the corresponding single spectra.

that couples to non-interacting conduction-band electrons and a Coulomb interaction $U$ between electrons on that level.

The effect of the Kondo resonance in the spectral function can be understood in analogy to other fields of physics, for example scattering of electrons at a potential well: the resonance causes an enhanced scattering amplitude and an energy-dependent phase shift of (quasi-) particles near the resonance energy.

In many-body formalism the measured LDOS is the imaginary part of the single-electron Green's function $G(\mathbf{x}, \mathbf{x}, \varepsilon)$. To calculate this quantity we use the Dyson equation, which connects the Green's function and hence the LDOS of the perturbed system to the
Green's function $G^{0}\left(\mathbf{x}, \mathbf{x}^{\prime}, \varepsilon\right)$ of the unperturbed conduction band electrons by a T-matrix $T\left(\mathbf{x}_{\mathrm{imp}}, \varepsilon\right)$. Approximating the impurity to be a point scatterer at position $\mathbf{x}_{\mathrm{imp}}$, the LDOS change is given by

$$
\Delta \operatorname{LDOS}(\mathbf{x}, \varepsilon)=-\frac{1}{\pi} \operatorname{Im}\left[G^{0}\left(\mathbf{x}, \mathbf{x}_{\mathrm{imp}}, \varepsilon\right) T\left(\mathbf{x}_{\mathrm{imp}}, \varepsilon\right) G^{0}\left(\mathbf{x}_{\mathrm{imp}}, \mathbf{x}, \varepsilon\right)\right](1)
$$

Calculating the band structure of copper using a linear combination of the atomic orbitals (LCAO) approach (Fig. 3a) and treating the surface as a potential step by fixing the continuity conditions for the wavefunctions we obtain the free Green's functions $G^{0}\left(\mathbf{x}, \mathbf{x}^{\prime}, \varepsilon\right)$ 
for copper (Fig. 3b and Supplementary Information). For the evaluation of the T-matrix we apply the numerical renormalization group $^{16}$ (NRG). At this point the imaginary part of the unperturbed Green's functions $G^{0}\left(\mathbf{x}_{\text {imp }}, \mathbf{x}_{\text {imp }}, \varepsilon\right)$ at the impurity position is needed to describe the states of the conduction band that couple to the impurity. Note that the presence of the surface leads to oscillations in this quantity (see Fig. 3c), which can be understood as a standing-wave pattern of electrons being reflected at the surface. The NRG calculations result in a Kondo resonance in the T-matrix near $\varepsilon_{\mathrm{F}}$ (Fig. $3 \mathrm{~d}$ ), with a resonance height and width showing similar oscillatory behaviour as seen in the unperturbed Green's functions $G^{0}\left(\mathbf{x}, \mathbf{x}^{\prime}, \varepsilon\right)$.

With the $T$-matrix obtained by the above procedure we calculate the LDOS change using equation(1). Values for energy and hybridization of the localized orbital were taken from $a b$ initio results ${ }^{17}$. The only free parameter left, the Coulomb interaction $U$, was adjusted by comparison to the experiment. The calculated LDOS sections (Fig. 3e) are in excellent agreement with the measured data (compare Fig. 2). The experimentally observed periodicity (compare a third- and seventh-layer impurity) is also found in the simulation.

The rich spatial and spectroscopic information of the measured interference patterns allow further investigation of the Kondo resonance. The resonance width is proportional to the Kondo temperature (see Supplementary Information). We fit the experimental data to a phenomenological form found by Frota (see discussion further down) for all lateral positions and a constant impurity depth. Averaging over all depths, we get a Kondo temperature $T_{\mathrm{K}}$ for Fe impurities of 32(16) K and for Co of 655(155) K. Results from macroscopic bulk measurements ${ }^{18}$ give similar values.

Investigating the Kondo temperature as a function of the lateral position for a constant impurity depth we found an unexpected variation of the resonance width. As an example, the fourthlayer Fe impurity (Fig. 1c) shows a variation of $T_{\mathrm{K}}(\mathrm{A})=29(9) \mathrm{K}$, $T_{\mathrm{K}}(\mathrm{B})=35(24) \mathrm{K}, T_{\mathrm{K}}(\mathrm{C})=42(18) \mathrm{K}$ and $T_{\mathrm{K}}(\mathrm{D})=44(26) \mathrm{K}$ from position A to $\mathrm{D}$. This behaviour is not included in our theory so far. One possible explanation employs the actual orbital structure of the $d$-level in Fe or Co, which leads to a dependence of the T-matrix on the propagation direction.

Going further, we investigate not only the long-range signature of the scattering amplitude but also its phase. During our analysis it turned out that the widely-used (complex) Lorentzian approximation of the Kondo resonance does not give the best description to both our experimental and theoretical data (see Supplementary Information). A Lorentzian for the T-matrix results in the often-used Fano line shapes ${ }^{19}$. Better fits were obtained by a phenomenological form found by Frota ${ }^{20,21}$. The scattering amplitude of the Kondo resonance decays much more weakly with energy than a Lorentzian, which can be viewed as a minor correction. More significantly, the shift of the scattering phase due to the Kondo resonance is strongly overestimated, as a Lorentzian always results in a phase shift of $\pi$. In the range of energies considered here, our NRG calculations give a phase shift of only $\pi / 2$, which is also described by the phenomenological form by Frota. As one can see in Fig. 1b, the phase shift measured in the experiment is also smaller than $\pi$.

The mapping of the scattering phase as a function of energy opens a new way to discriminate between single-particle resonances and Kondo scattering. Our approach allows further investigations of the properties of magnetic impurities, such as the splitting of the resonance by an applied magnetic field. This was recently shown on adsorbed atoms $\mathrm{s}^{22}$ and should also affect the scattering behaviour of subsurface impurities. With available magnetic fields, manganese may be a good candidate for such an experiment. As we observe a long-range Kondo signature, this opens a new way to examine the interaction between two or more Kondo atoms with each other, or the effect of an interface in real space. Finally, this observation may give an insight into one of the most controversial terms discussed in Kondo physics: the meaning and size of the 'Kondo cloud'. With the long-range LDOS signatures of buried impurities, charge density oscillations are accessible and the term 'Kondo cloud' may be defined in a way that is observable and consistent with experiments.

Received 8 March 2010; accepted 4 November 2010;

published online 23 January 2011; corrected online

9 January 2012

\section{References}

1. Hewson, A. C. The Kondo Problem to Heavy Fermions (Cambridge Univ. Press, 1993).

2. Li, J., Schneider, W-D., Berndt, R. \& Delley, B. Kondo scattering observed at a single magnetic impurity. Phys. Rev. Lett. 80, 2893-2896 (1998).

3. Madhavan, V., Chen, W., Jamneala, T., Crommie, M. F. \& Wingreen, N. S. Tunneling into a single magnetic atom: Spectroscopic evidence of the Kondo resonance. Science 280, 567-569 (1998).

4. Manoharan, H. C., Lutz, C. P. \& Eigler, D. M. Quantum mirages formed by coherent projection of electronic structure. Nature 403, 512-515 (2000).

5. Quaas, N., Wenderoth, M., Weismann, A., Ulbrich, R. G. \& Schönhammer, K. Kondo resonance of single Co atoms embedded in $\mathrm{Cu}(111)$. Phys. Rev. B 69, 201103 (2004).

6. Zhao, A. et al. Controlling the Kondo effect of an adsorbed magnetic ion through its chemical bonding. Science 309, 1542-1544 (2005).

7. Iancu, V., Deshpande, A. \& Hla, S-W. Manipulating Kondo temperature via single molecule switching. Nano Lett. 6, 820-823 (2006).

8. Néel, N. et al. Conductance and Kondo effect in a controlled single-atom contact. Phys. Rev. Lett. 98, 016801 (2007).

9. Néel, N. et al. Controlling the Kondo effect in $\mathrm{CoCu}_{n}$ clusters atom by atom Phys. Rev. Lett. 101, 266803 (2008).

10. Patthey, F., Delley, B., Schneider, W-D. \& Baer, Y. Low-energy excitations in alpha- and gamma-Ce observed by photoemission. Phys. Rev. Lett. 55, 1518-1521 (1985).

11. Ehm, D. et al. High-resolution photoemission study on low- $t_{\mathrm{k}}$ Ce systems: Kondo resonance, crystal field structures, and their temperature dependence. Phys. Rev. B 76, 045117 (2007).

12. Újsághy, O., Kroha, J., Szunyogh, L. \& Zawadowski, A. Theory of the Fano resonance in the stm tunneling density of states due to a single Kondo impurity. Phys. Rev. Lett. 85, 2557-2560 (2000).

13. Ternes, M., Heinrich, A. J. \& Schneider, W-D. Spectroscopic manifestations of the Kondo effect on single adatoms. J. Phys. Condens. Matter 5, 053001 (2009).

14. Weismann, A. et al. Seeing the Fermi surface in real space by nanoscale electron focusing. Science 323, 1190-1193 (2009).

15. Lounis, S. et al. Theory of real space imaging of Fermi surfaces. Preprint at http://arxiv.org/abs/1010.2112 (2010).

16. Bulla, R., Costi, T. A. \& Pruschke, T. Numerical renormalization group method for quantum impurity systems. Rev. Mod. Phys. 80, 395-450 (2008).

17. Lin, C-Y., Castro Neto, A. H. \& Jones, B. A. First-principles calculation of the single impurity surface Kondo resonance. Phys. Rev. Lett. 97, 156102 (2006).

18. Daybell, M. D. \& Steyert, W. A. Localized magnetic impurity states in metals: Some experimental relationships. Rev. Mod. Phys. 40, 380-389 (1968).

19. Fano, U. Effects of configuration interaction on intensities and phase shifts. Phys. Rev. 124, 1866-1878 (1961).

20. Frota, H. O. \& Oliveira, L. N. Photoemission spectroscopy for the spin-degenerate Anderson model. Phys. Rev. B 33, 7871-7874 (1986).

21. Frota, H. O. Shape of the Kondo resonance. Phys. Rev. B 45, 1096-1099 (1992).

22. Otte, A. F. et al. The role of magnetic anisotropy in the Kondo effect. Nature Phys. 4, 847-850 (2008).

\section{Acknowledgements}

This work was supported by the Deutsche Forschungsgemeinschaft through SFB 602 Project A3.

\section{Author contributions}

M.W., A.W. and R.G.U. planned the experiments. H.P. and A.W. carried out the experiments and the data analysis. A.W. carried out the band-structure (LCAO) and LDOS calculations. P.E.D. did the NRG calculations with the code provided by R.P. and T.P. H.P. and P.E.D. wrote the manuscript. All authors discussed the results and commented on the manuscript.

\section{Additional information}

The authors declare no competing financial interests. Supplementary information accompanies this paper on www.nature.com/naturephysics. Reprints and permissions information is available online at http://npg.nature.com/reprintsandpermissions. Correspondence and requests for materials should be addressed to M.W. 
CORRIGENDUM

\section{Long-range Kondo signature of a single magnetic impurity}

Henning Prüser, Martin Wenderoth, Piet E. Dargel, Alexander Weismann, Robert Peters, Thomas Pruschke and Rainer G. Ulbrich

Nature Physics 7, 203-206 (2011); published online 23 January 2011; corrected after print 9 January 2012.

In the version of this Letter originally published, in Fig. 3a the $y$-axis units should have been 'eV'. This error has been corrected in the HTML and PDF versions of the Letter. 
CORRIGENDUM

\section{Long-range Kondo signature of a single magnetic impurity}

Henning Prüser, Martin Wenderoth, Piet E. Dargel, Alexander Weismann, Robert Peters, Thomas Pruschke and Rainer G. Ulbrich

Nature Physics 7, 203-206 (2011); published online 23 January 2011; corrected after print 9 January 2012.

In the version of this Letter originally published, in Fig. 3a the $y$-axis units should have been 'eV'. This error has been corrected in the HTML and PDF versions of the Letter. 